International Mathematical Forum, 3, 2008, no. 44, 2181 - 2189

\title{
Aggregation Functions:
}

\section{A Multivariate Approach Using Copulae}

\author{
Marta Cardin \\ Dept. of Applied Mathematics \\ University of Venice \\ mcardin@unive.it \\ Maddalena Manzi \\ Dept. of Mathematics \\ University of Padua \\ mmanzi@math.unipd.it
}

\begin{abstract}
In this paper we present the extension of the copula approach to aggregation functions. In fact we want to focus on a class of aggregation functions and present them in the multi linear form with marginal copulae. Moreover we will define also the joint aggregation density function.
\end{abstract}

Mathematics Subject Classification: 90B50, 91B82, 60A10, 60E15

Keywords: Aggregation function, $n$-increasing function, copula, joint aggregation density function, absolutely continuity

\section{Introduction}

In many decision situations, we are faced with multiple and conflicting attributes, that is with the problem of aggregating a collection of numerical readings to obtain a typical value. So, aggregation functions are used in multicriteria decision making problems to obtain a global score, but also in many different domains. We give some formal definitions related to the problem of aggregation and in particular we present a new unified approach to copulabased modeling and characterizations of aggregation functions. The concept of copula introduced by Sklar in 1959 is now common in the statistical literature, but only recently its potential for applications has become clear. Copulae 
permit to represent joint distribution functions by splitting the marginal behavior, embedded in the marginal distributions, from the dependence captured by the copula itself. So, the natural application of this function in the problem of modeling interaction between attributes is really an interesting question in the theory of aggregation functions.

The paper is structured as follows. In the next section we introduce briefly the general background of aggregation functions. After a brief overview about some properties of copulae, we present the copula approach for studying aggregation problems, considering the case $n=4$. In fact we have already studied the bivariate and trivariate cases. Now we extend this problem to $n$ dimensions, considering in the last section some perspectives for future developments.

\section{Definitions and properties}

The aggregation operators are mathematical objects that have the function of reducing a set of numbers into a unique representative number.

For example, the arithmetic mean as an aggregation function is defined by

$$
A M\left(x_{1}, \ldots, x_{n}\right)=\frac{1}{n} \sum_{i=1}^{n} x_{i} .
$$

We introduce some properties which could be desirable for the aggregation of criteria.

If we consider the behavior of the aggregation in the best and in the worst case we expect that an aggregation satisfies the following boundary conditions :

$$
A(0, \ldots, 0)=0 \quad \text { and } \quad A(1, \ldots, 1)=1
$$

These conditions mean that if we observe only completely bad (or satisfactory) criteria the total aggregation has to be completely bad (or satisfactory). We consider aggregation functions that satisfy the boundary conditions.

Increasingness is another property, which is often required for aggregation and commonly accepted for functions used to aggregate preferences.

So, we can define an aggregation operator as a function

$$
A:[0,1]^{n} \rightarrow[0,1]
$$

that satisfies:

- $A(0, \ldots, 0)=0$ and $A(1, \ldots, 1)=1$

- $A\left(x_{1}, \ldots, x_{n}\right) \leq A\left(y_{1}, \ldots, y_{n}\right)$ if $\left(x_{1}, \ldots, x_{n}\right) \leq\left(y_{1}, \ldots, y_{n}\right)$ 
Associativity is also an interesting property for aggregation functions. The associativity property concerns the " clustering " character of an aggregation function. The properties that could be required for an aggregation function are generally based on natural considerations corresponding to the idea of an aggregated value. In deciding on the form of the aggregation operator, another elementary mathematical property can be continuity in the usual sense. We extend our analysis to the continuous case and in particular we give the notions of absolute continuity for multivariate functions.

We will refer to $\partial f$ as the density of $f$.

Definition 2.1 The density of an absolutely continuous function $f$, denoted $\partial f$, is any real valued function on $[a, b]^{n}$ such that $\int_{R} \partial f=\Delta_{R} f$ for any $n$ dimensional rectangle $R \subseteq[a, b]^{n}$.

So a function $f$ is called absolutely continuous if, when considered as a joint cdf, it has a joint density given by $\partial^{n} f^{n}\left(x_{1}, \ldots, x_{n}\right) / \partial x_{1}, \partial x_{2}, \ldots, \partial x_{n}$.

When modeling multivariate distributions, one has to take into account the effects of the marginal distributions as well as the dependence between them. This can be achieved by using the copula approach, which allows to deal with the margins and the dependence structure separately. Although almost 50 years old copulae have only recently been applied in a variety of areas. Now we briefly introduce some definitions and properties that lie within the scope of this article.

Definition 2.2 A function $f: \mathrm{R}^{n} \rightarrow \mathrm{R}$ is n-increasing if $V_{f}(B) \geq 0$ for all $n$-box $B=\left[a_{1}, b_{1}\right] \times\left[a_{2}, b_{2}\right] \times \ldots \times\left[a_{n}, b_{n}\right] \subseteq[0,1]^{n}$ with $a_{i} \leq b_{i}, i=1,2, \ldots, n$

If $f$ has nth-order derivatives, n-increasing is equivalent to $\frac{\partial^{n}}{\partial X_{1} \partial X_{2} \ldots \partial X_{n}} f \geq 0$. This definition is the multivariate extension of the concept of "increasing" for a univariate function when we interpret "increasing" as "increasing as a distribution function".

An $n$-copula is the restriction to the unit cube $[0,1]^{n}$ of a multivariate cumulative distribution function, whose marginals are uniform on $[0,1]$. More precisely, an $n$-copula is a function $C:[0,1]^{n} \rightarrow[0,1]$ that satisfies:

(a) $C(\mathbf{x})=0$ if $x_{i}=0$ for any $i=1, \ldots, n$, that is $C$ is grounded;

(b) $C(\mathbf{x})=x_{i}$ if all coordinates of $\mathbf{x}$ are 1 except $x_{i}$, that is $C$ has uniform one-dimensional marginals;

(c) $\mathrm{C}$ is n-increasing. 
Conditions (a) and (b) are known as boundary conditions whereas condition (c) is known as monotonicity.

Various properties of copulae have been studied in literature, but most part of the research concentrates on the bivariate case, since multivariate extensions are generally not easily to be done. Moreover, for any $n$-copula:

$$
W\left(x_{1}, \ldots, x_{n}\right) \leq C\left(x_{1}, \ldots, x_{n}\right) \leq M\left(x_{1}, \ldots, x_{n}\right) .
$$

The upper function $M$ is an $n$-copula for any $n \in \mathrm{N}$, the lower function $W$ is not an $n$-copula for any $n>2$.

Now we recall also Sklar's theorem:

Theorem 2.3 (Sklar 72) If $X_{1}, \ldots, X_{n}$ are random variables defined on a common probability space, with the one-dimensional cdf's $F_{X_{k}}\left(x_{k}\right)=P\left(X_{k} \leq\right.$ $\left.x_{k}\right)$ and the joint $c d f F_{X_{1}, \ldots, X_{n}}\left(x_{1}, \ldots, x_{n}\right)=P\left(X_{1} \leq x_{1}, \ldots, X_{n} \leq x_{n}\right)$, then there exists an $n$-dimensional copula $C_{X_{1}, \ldots, X_{n}}\left(u_{1}, \ldots, u_{n}\right)$ such that $F_{X_{1}, \ldots, X_{n}}\left(x_{1}, \ldots, x_{n}\right)=C_{X_{1}, \ldots, X_{n}}\left(F_{X_{1}}\left(x_{1}\right), \ldots, F_{X_{n}}\left(x_{n}\right)\right)$ for all $x_{k} \in \mathbf{R}$, $k=1, \ldots, n$.

A rigorous mathematical description of copulae and their features is available in [11].

\section{The general case}

If we consider $A\left(x_{1}, x_{2}, x_{3}, x_{4}\right)$, we observe that, by using the previous observation,

$$
\begin{gathered}
A\left(x_{1}, x_{2}, x_{3}, x_{4}\right)=A\left(x_{1}, x_{2}, x_{3}, 0\right)+A\left(x_{1}, 0, x_{3}, x_{4}\right)+A\left(x_{1}, x_{2}, 0, x_{4}\right)+ \\
+A\left(0, x_{2}, x_{3}, x_{4}\right)-A\left(x_{1}, 0,0, x_{4}\right)-A\left(x_{1}, x_{2}, 0,0\right)-A\left(x_{1}, 0, x_{3}, 0\right)+ \\
-A\left(0, x_{2}, x_{3}, 0\right)-A\left(0, x_{2}, 0, x_{4}\right)-A\left(0,0, x_{3}, x_{4}\right)+A\left(x_{1}, 0,0,0\right)+A\left(0, x_{2}, 0,0\right)+ \\
+A\left(0,0, x_{3}, 0\right)+A\left(0,0,0, x_{4}\right)+H\left(x_{1}, x_{2}, x_{3}, x_{4}\right) .
\end{gathered}
$$

By using and iterating the previous decomposition, we have the following situation:

1. Three bivariate copulae $C\left(F_{i}, F_{j}\right) \forall i=1,2,3 \quad j>i$, with $F_{i}=F_{i}\left(x_{i}\right)$.

2. One trivariate copula $C\left(F_{i}, F_{j}, F_{l}\right) \forall i=1,2,3 \quad j>i \quad l>j$.

In fact the trivariate copulae go out only from the respective trivariate aggregation functions, while, about the bivariate ones, we have that, for the same $i$ and $j$ only one goes out from the respective bivariate aggregation 
function. We have the other two thanks to the trivariate aggregation functions. For example, we have three $C\left(F_{1}, F_{2}\right)$ : one from $A\left(x_{1}, x_{2}, 0,0\right)$, that is $k^{34} C^{34}\left(F_{1}^{34}, F_{2}^{34}\right)$, another from $A\left(x_{1}, x_{2}, x_{3}, 0\right)$, that is $k^{4} C^{4}\left(F_{1}^{4}, F_{2}^{4}\right)$ and the last from $A\left(x_{1}, x_{2}, 0, x_{4}\right)$, that is $k^{3} C^{3}\left(F_{1}^{3}, F_{2}^{3}\right)$.

So we can write the following decomposition:

$$
\begin{aligned}
& A\left(x_{1}, x_{2}, x_{3}, x_{4}\right)=\sum_{i=1}^{4} A_{i}\left(x_{i}\right)+\sum_{\substack{\{h, k\} \\
h=1}}^{4} \alpha^{\{\overline{\{h, k\}}=0\}} C^{\{\overline{\{h, k\}}=0\}}\left(F_{h}^{\{\overline{\{h, k\}}=0\}}, F_{k}^{\{\overline{\{h, k\}}=0\}}\right)+ \\
& +\sum_{\substack{\{i, j, l\} \\
i=1}}^{4} \alpha^{\{\overline{\{i, j, l\}}=0\}} C^{\{\overline{\{i, j, l\}}=0\}}\left(F_{i}^{\{\overline{\{i, j, l\}}=0\}}, F_{j}^{\{\overline{\{i, j, l\}}=0\}}, F_{l}^{\{\overline{\{i, j, l\}}=0\}}\right)+\alpha C\left(F_{1}, F_{2}, F_{3}, F_{4}\right) .
\end{aligned}
$$

where $\{\overline{\{h, k\}}=0\}$ is the set of complementary attributes of $\left\{x_{h}, x_{k}\right\}$, when, at least one of them is equal to zero and $\{\overline{\{i, j, l\}}=0\}$ is the set of complementary attributes of $\left\{x_{i}, x_{j}, x_{l}\right\}$, when, at least one of them is equal to zero too.

Now we can prove the general result.

Theorem 3.1 (General case) If $A$ is absolutely continuous aggregation function, there exist $n$ increasing and continuous functions $A_{i}\left(x_{i}\right), n(n-1)+$ $\left(\begin{array}{l}n \\ 2\end{array}\right)$ bivariate copulae $C, n(n-1)(n-2) \ldots(n-k)+\left(\begin{array}{l}n \\ k\end{array}\right) k$-copulae... and an n-copula, such that

$$
\begin{aligned}
A\left(x_{1}, \ldots, x_{n}\right) & =\sum_{i=1}^{n} A_{i}\left(x_{i}\right)+\sum_{\substack{\{r, s\} \\
r=1}}^{n} \bar{\alpha}_{r, s} C\left(\bar{F}_{r, s}\right)+\ldots \\
\ldots & +\sum_{\substack{\{r, s, \ldots, t\} \\
r=1}}^{n} \bar{\alpha}_{r, s, \ldots, t} C\left(\bar{F}_{r, s, \ldots, t}\right)+\ldots+\alpha C\left(F_{1}, \ldots, F_{n}\right),
\end{aligned}
$$

with $A_{i}\left(x_{i}\right)=A\left(0, \ldots, x_{i}, \ldots, 0\right)$,

$\bar{F}_{r, s, \ldots, t}=\left(F_{r}^{\{\overline{\{r, s, \ldots, t\}}=0\}}, F_{s}^{\{\overline{\{r, s, \ldots, t\}}=0\}}, \ldots, F_{t}^{\{\overline{\{r, s, \ldots, t\}}=0\}}\right)$ and $\{r, s, \ldots, t\}$ is the set of the attributes that are not equal to zero.

Proof.

We can prove this by induction:

$n=2$ and $n=3$ are right and $n-1 \Rightarrow n$. So our inductive hypothesis is:

$$
\begin{aligned}
A\left(x_{1}, \ldots, x_{n-1}\right) & =\sum_{i=1}^{n-1} A_{i}\left(x_{i}\right)+\sum_{\substack{\{r, s\} \\
r=1}}^{n-1} \bar{\alpha}_{r, s} C\left(\bar{F}_{r, s}\right)+\ldots \\
& \ldots+\sum_{\substack{\{r, s, \ldots, t\} \\
r=1}}^{n-1} \bar{\alpha}_{r, s, \ldots, t} C\left(\bar{F}_{r, s, \ldots, t}\right)+\ldots+\alpha C\left(F_{1}, \ldots, F_{n-1}\right)
\end{aligned}
$$


and we can apply this to the previous formula, but first we define $A\left(x_{1}, \ldots, x_{j}, \ldots, x_{n}\right)_{\mid x_{j}=0}=A\left(\mathbf{x}_{\circ}^{j}\right)$, where $\mathbf{x}_{\circ}^{j}$ is the set of complementary attributes of $x_{j}$, with $x_{j}=0$ and similarly $\mathbf{x}_{\circ}^{\{j, l, \ldots, m\}}$ as the set of complementary attributes of $x_{j}=x_{l}=\ldots=x_{m}=0$.

So, we have:

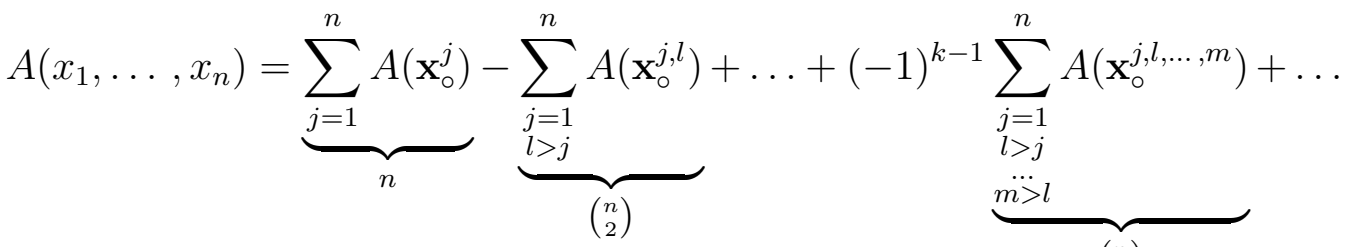

$$
\begin{aligned}
& \left(\begin{array}{l}
n \\
k
\end{array}\right) \\
& \ldots+H\left(F_{1}, \ldots, F_{n}\right) .
\end{aligned}
$$

Then we have for our hypothesis:

$$
\begin{aligned}
A\left(\mathbf{x}_{\circ}^{j}\right) & =A\left(x_{1}, \ldots, x_{j}, \ldots, x_{n}\right)_{\mid x_{j}=0}=A\left(x_{1}, \ldots, x_{n-1}\right)=\sum_{\substack{i=1 \\
i \neq j}}^{n-1} A_{i}\left(x_{i}\right)+ \\
& +\sum_{\substack{\{r, s\} \\
r=1}}^{n-1} \bar{\alpha}_{r, s} C\left(\bar{F}_{r, s}\right)+\ldots+\sum_{\substack{\{r, s, \ldots, t\} \\
r=1}}^{n-1} \bar{\alpha}_{r, s, \ldots, t} C\left(\bar{F}_{r, s, \ldots, t}\right)+\ldots \\
& \ldots+\alpha^{j} C\left(F_{1}, \ldots, F_{n-1}\right),
\end{aligned}
$$

$\forall j$. Similarly,

$$
\begin{aligned}
A\left(\mathbf{x}_{\circ}^{j, l}\right) & =\sum_{\substack{i=1 \\
i \neq(j, l) \\
l>j}}^{n-2} A_{i}\left(x_{i}\right)+\sum_{\substack{\{r, s\} \\
r=1}}^{n-2} \bar{\alpha}_{r, s} C\left(\bar{F}_{r, s}\right)+\ldots \\
& \ldots+\sum_{\substack{\{r, s, \ldots, t\} \\
r=1}}^{n-2} \bar{\alpha}_{r, s, \ldots, t} C\left(\bar{F}_{r, s, \ldots, t}\right)+\ldots+\alpha^{j, l} C\left(F_{1}, \ldots, F_{n-2}\right),
\end{aligned}
$$

$\forall j, l$ and

$$
\begin{aligned}
A\left(\mathbf{x}_{\circ}^{j, l, \ldots, m}\right)= & \sum_{\substack{i=1 \\
i \neq j, j, \ldots, m) \\
l, l, j \\
l \ldots j \\
m>l}}^{n-k} A_{i}\left(x_{i}\right)+\sum_{\substack{\{r, s\} \\
r=1}}^{n-k} \bar{\alpha}_{r, s} C\left(\bar{F}_{r, s}\right)+\ldots \\
& \ldots+\sum_{\substack{\{r, s, \ldots, t\} \\
r=1}}^{n-k} \bar{\alpha}_{r, s, \ldots, t} C\left(\bar{F}_{r, s, \ldots, t}\right)+\ldots+\alpha^{j, l, \ldots, m} C\left(F_{1}, \ldots, F_{n-k}\right),
\end{aligned}
$$


$\forall j, l, m$. So:

$$
\begin{aligned}
& A\left(x_{1}, \ldots, x_{n}\right)=\sum_{j=1}^{n} A\left(\mathbf{x}_{\circ}^{j}\right)-\sum_{\substack{j=1 \\
l>j}}^{n} A\left(\mathbf{x}_{\circ}^{j, l}\right)+\ldots+(-1)^{k-1} \sum_{\substack{j=1 \\
l>j \\
m>l}}^{n} A\left(\mathbf{x}_{\circ}^{j, l, \ldots, m}\right)+\ldots \\
& \ldots+H\left(F_{1}, \ldots, F_{n}\right)=\sum_{j=1}^{n} \sum_{\substack{i=1 \\
i \neq j}}^{n-1} A_{i}\left(x_{i}\right)+\sum_{j=1}^{n} \sum_{\substack{\{r, s\} \\
r=1}}^{n-1} \bar{\alpha}_{r, s} C\left(\bar{F}_{r, s}\right)+\ldots \\
& \ldots+\sum_{j=1}^{n} \sum_{\substack{\{r, s, \ldots, t\} \\
r=1}}^{n-1} \bar{\alpha}_{r, s, \ldots, t} C\left(\bar{F}_{r, s, \ldots, t}\right)+\ldots+\sum_{j=1}^{n} \alpha^{j} C\left(F_{1}, \ldots, F_{n-1}\right)- \\
& -\sum_{\substack{j=1 \\
l>j}}^{n} \sum_{\substack{i=1 \\
i \neq(j, l) \\
l>j}}^{n-2} A_{i}\left(x_{i}\right)-\sum_{\substack{j=1 \\
l>j}}^{n} \sum_{\substack{\{r, s\} \\
r=1}}^{n-2} \bar{\alpha}_{r, s} C\left(\bar{F}_{r, s}\right)-\ldots \\
& \ldots-\sum_{\substack{j=1 \\
l>j}}^{n} \sum_{\substack{\{r, s, \ldots, t\} \\
r=1}}^{n-2} \bar{\alpha}_{r, s, \ldots, t} C\left(\bar{F}_{r, s, \ldots, t}\right)-\ldots-\sum_{\substack{j=1 \\
l>j}}^{n} \alpha^{j, l} C\left(F_{1}, \ldots, F_{n-2}\right)+\ldots \\
& \ldots+(-1)^{k-1} \sum_{\substack{j=1 \\
l>j \\
m>l}}^{n} \sum_{\substack{i=1 \\
m>(j, l, \ldots, m) \\
l>j \\
\ldots>m>l}}^{n-k} A_{i}\left(x_{i}\right)+(-1)^{k-1} \sum_{\substack{j=1 \\
l>j \\
m>l \\
m>l}}^{n} \sum_{\substack{m, s\} \\
m>l}}^{n-k} \bar{\alpha}_{r, s} C\left(\bar{F}_{r, s}\right)+\ldots \\
& \ldots+(-1)^{k-1} \sum_{\substack{j=1 \\
l>j \\
m>l}}^{n} \sum_{\substack{\{r, s, \ldots, t\} \\
r=1}}^{n-k} \bar{\alpha}_{r, s, \ldots, t} C\left(\bar{F}_{r, s, \ldots, t}\right)+\ldots \\
& \ldots+(-1)^{k-1} \sum_{\substack{j=1 \\
l>j \\
m>l}}^{n} \alpha^{j, l, \ldots, m} C\left(F_{1}, \ldots, F_{n-k}\right)+\ldots+H\left(F_{1}, \ldots, F_{n}\right)
\end{aligned}
$$

and so, by computing this expression, we have our thesis.

\section{Concluding remarks}

In this work we have analyzed the copula approach to aggregation functions, continuing the study of our previous work [2]. The decomposition of a multivariate distribution function between its marginal distribution functions and 
its dependence structure facilitates its analysis and understanding. In this paper, a new method to construct multivariate aggregation functions is introduced. In fact our method can be used in the other sense to prove that the sum of several copulae in a linear combination is an aggregation function. This approach has been generalized to $n$ dimensions, studying $n$-increasing functions and in particular those ones which are $n$-copulae. However, this generalization remains an open problem in the application to discrete copulae, quasi-copulae and in particular with respect to no finite dimensional copulae.

\section{References}

[1] T. Calvo, G. Mayor, R. Mesiar (Eds.), (2002). Aggregation operators. New Trends and Applications, Physica-Verlag, Heidelberg.

[2] M. Cardin, M. Manzi (2007). "Supermodular binary aggregation operators and copulae", SSRN-id 1024444

[3] F. Durante R. Mesiar, P.L. Papini, C. Sempi (2007). "2-increasing binary aggregation operators", Infor.Sciences. 177, 111-129.

[4] F. Durante, R. Mesiar, C. Sempi (2006). "On a family of copulas constructed from the diagonal section", Soft Computing 10, 490-494.

[5] Hobson, E. W. (1927). "The Theory of Functions of a Real Variable and the Theory of Fourier's Series (3rd ed.)", Cambridge University Press 1. Republished by Harren Press (1950).

[6] H.Joe (1997). Multivariate Models and Dependence Concepts, Chapman \& Hall, London.

[7] G. Mayor, J. Suñer, J. Torrens. (2005). "Copula-Like Operations on Finite Settings", IEEE Transactions on fuzzy systems, 13, n.4, 468-476.

[8] E. P. Klement, R. Mesiar, E. Pap (2002). "Invariant copulas", Kybernetika (Prague), 38, 275-285.

[9] Mesiar R., Saminger S. (2004). "Domination of ordered weighted averaging operators over t-norms", Soft Computing 8, 562-570.

[10] Morillas M. P. (2005) "A characterization of absolutely monotonic $(\Delta)$ functions of a fixed order", Publications de l'institut mathématique, Nouvelle série, tome $\mathbf{7 8 ( 9 2 ) , ~ 9 3 - 1 0 5 . ~}$ 
[11] Roger B. Nelsen (1999). An Introduction to Copulas, in: Lecture Notes in Statistics, Vol. 139, Springer, New York.

[12] J. Von Neumann (1950). Functional operators, Volume I: Measures and integrals, Princeton University Press, Princeton, NJ.

Received: May 122008 